\title{
Effects of Mulberry Fruit Powder in Animal Model of Stroke
}

\author{
${ }^{1}$ Pratchaya Kaewkaen, ${ }^{2,3}$ Terdthai Tong-Un, ${ }^{2,3}$ Jintanaporn Wattanathorn, \\ ${ }^{2,3}$ Supaporn Muchimapura, ${ }^{4}$ Wiroje Kaewrueng and ${ }^{4}$ Sathaporn Wongcharoenwanakit \\ ${ }^{1}$ Department of Physiology and Graduate School (Neuroscience Program), \\ Faculty of Medicine, Khon Kaen University, Thailand 40002, Thailand \\ ${ }^{2}$ Department of Physiology, \\ Faculty of Medicine, Khon Kaen University, Thailand 40002, Thailand \\ ${ }^{3}$ Integrative Complimentary Alternative Medicine \\ Research Group, Khon Kaen University, Thailand 40002, Thailand \\ ${ }^{4}$ Department of Sericulture, \\ Ministry of Agriculture and Cooperatives, Bangkok, Thailand 10900, Thailand
}

Received 2012-03-24, Revised 2012-06-20; Accepted 2012-08-15

\begin{abstract}
Stroke is the leading cause of death and the important cause of disability worldwide. Presently, the effectiveness of therapeutic strategy is still very limited. Based on the role neuroprotective effect of substance possessing antioxidant effect previously reported, we hypothesized mulberry fruit a substance possessing anti-oxidant activity, might be able to protect against cognitive impairment induced by stroke or cerebral ischemia. Male Wistar rats were orally given mulberry fruit powder at doses of 2,10 and $50 \mathrm{mg}$ $\mathrm{kg}^{-1} \mathrm{BW}$ at a periods of 7 days before and 21 days after induced Middle Cerebral Artery Occlusion (MCAO). The animals were determined spatial memory using Morris water maze test, motor performance was determined using neurological score and the sensory performance was also determined using hot plate test every 7 days before and after the operation until the end of experiment. In addition, the density of survival neurons was determined using cresyl violet stain. The results showed that the significant improvement of mulberry fruits in healthy condition after single dose and 7 days of MCAO at dose 2 and 10 $\mathrm{mg} \mathrm{kg}^{-1} \mathrm{BW}$. In cognitive deficit condition, mulberry fruits can exert neuroprotective at dose 10 and $50 \mathrm{mg}$ $\mathrm{kg}^{-1} \mathrm{BW}$ after 21 days MCAO. Moreover, the significant change of sensory recovery was observed at 50 $\mathrm{mg} \mathrm{kg}$ BW after MCAO 7 days. The mulberry fruits significantly improved the density of survival neurons in CA2 and CA3 of hippocampus after 21 days of MCAO. All results in this study suggested that mulberry fruit had high potential to be developed as novel preventive and therapeutic strategy against stroke condition. However, precise underlying mechanism is still required further investigation.
\end{abstract}

Keywords: Mulberry Fruit, Cognitive Enhancing, Animal Model Of Stroke, Stroke Condition, Precise Underlying, Cognitive Deficit, Middle Cerebral Artery Occlusion (MCAO)

\section{INTRODUCTION}

Stroke has been recognized as a leading cause of death and disability in many countries. At present, millions of people were suffering from stroke worldwide. Despite the enormity of the problem, there is currently no approved therapy that can reduce infarct size or neurological disability. One of the approaches that can be used in limiting the neurological damage after stroke is the use of prophylactic treatment in patients with a high-risk of stroke. Therefore, stroke prevention is also very important.
The cognitive disturbance commonly found in stroke. This condition can be varied from less to very serious condition (Brainin et al., 2007). In general, the severity of cognitive impairment may depend on the location and magnitude of cerebral arteries injury. Moreover, the clinical manifestations consist of memory loss, confusion, executive dysfunction and impairment of motor control (Roman et al., 2004). Especially, the dorsal hippocampus damage had been identified as one of the main causes of behavioral decline in animal model of cognitive impairment (Park et al., 2000). In old rats, 
chronic brain hypoperfusion produces deficits in spatial learning and memory (Cada et al., 2000).

Presently, many strategies have been developed to optimize learning and memory including exercise, dietary therapy, herbal therapy, pharmaceutical drugs and advanced medical device. Among various strategies, dietary therapy such as functional food has gained much concentration. Functional foods are not pills or capsules but they are consumed as part-of a normal everyday diet. The beneficial effect of functional food appears to relate to its bioactive ingredient such as substances containing antioxidant (Raghuveer and Tandon, 2009). Mulberry or Morus alba, a plant in a family of Moracaea, is widely grown in many regions of the world, predominantly in eastern, southern and southeastern Asia. Various parts of mulberry have been used in the Oriental Medicine for numerous medicinal purposes including anti-diabetes, anti-inflammatory, diuretic, antitussive and antipyretic. Mulberry fruits has been previously used as both a tonic and sedative. Recently, mulberry fruits are reported to contain high concentration of anthocyanin (Dugo et al., 2001), a substance possessing potent antioxidant activity and numerous therapeutic effects (Dugo et al., 2001; Mitcheva et al., 1993). Abundant lines of evidence suggested that anthocyanin could reduce cardiovascular risk, improve inflammation and protect against chemical toxicity (Lazze et al., 2004; Meyer et al., 2001; Tsuda et al., 1998) and cerebral ischemic damage (Kang et al., 2006). Moreover, there is no available toxic information about mulberry fruits until now.

Based on the role neuroprotective effect of substance possessing antioxidant effect previously reported, we hypothesized mulberry fruit a substance possessing anti-oxidant activity, might be able to protect against cognitive impairment induced by stroke or cerebral ischemia. This study was conducted to investigate the effect of mulberry fruit on spatial memory and neurons density in hippocampus area of animal model of focal cerebral ischemia.

\section{MATERIALS AND METHODS}

\subsection{Plant Material and Preparation}

Animals: Adult male Wistar rats (300-350 g, 8 weeks old) were obtained from National Laboratory Animal Center, Salaya, Nakorn Pathom and were housed in group of 5 per cage in standard metal cages at $22 \pm 2{ }^{\circ} \mathrm{C}$ on 12:12 h light-dark cycle. All animals were given access to food and water ad libitum. The experiments were performed to minimize animal suffering in accordance with the internationally accepted principles for laboratory use and care of European Community (EEC directive of 1986; 86/609/EEC). The experimental protocols were approved by the Institutional Animal Care and Use Committee (AEKKU 1/2552).

\subsection{Drugs}

Mulberry fruits powder from The Queen Sirikit Department of Seri culture, Ministry of Agriculture and Cooperative, Thailand. Both Donepezil hydrochloride (Aricept ${ }^{\circledR}$ ), a standard drug used for mild cognitive impairment treatment and vitamin $\mathrm{C}$, an antioxidant possessing the neuroprotective and cognitive enhancing effects were used as positive controls in this study. Distilled Water (DW) was used as vehicle throughout the study. All administered substances were freshly prepared.

\subsection{Plant Preparation}

All mulberry fruits used in this study is prepared and provided by The Queen Sirikit Department of Seri Culture, Thailand. Mulberry fruits were collected from the Queen Sirikit Seri Culture Center Udon Thani. All berries were picked at the commercially ripen stage and selected according to uniformity color. Then, the fruits were dried at $70^{\circ} \mathrm{C}$ for 4 days and grounded to powder.

\subsection{Experimental Protocol}

All rats were randomly divided into 6 groups. Each group contained 6 rats.

\subsection{Group 1}

Vehicle treated group. The animals in this group were treated with distilled water

\subsection{Group 2-3}

Positive control treated group. The positive control group was treated with the standard drugs used for treating the related the condition. Vitamin C, a wellknown antioxidant, was orally administered at dose of $250 \mathrm{mg} \mathrm{kg} \mathrm{BW}^{-1}$. In the determination of cognitive function, the positive control group was treated with Donepezil (Aricept ${ }^{\circledR}$, a cholinesterase inhibitor) at dose of $1 \mathrm{mg} \mathrm{kg} \mathrm{BW}{ }^{-1}$

\subsection{Group 4-6}

Mulberry fruits powder treated group. The animals in group 4-6 were treated with the Mulberry fruits powder rat various doses ranging from 2,10 and $50 \mathrm{mg} \mathrm{kg} \mathrm{BW}^{-1}$ respectively via oral route administration for a week once daily throughout the experimental period and three weeks after induce middle cerebral artery occlusion.

All animals were treated with vehicle, positive control or mulberry fruits extract at a period of 7 days before and 21 days after right Middle Cerebral Artery Occlusion (MCAO). 


\subsection{Surgical Procedure to Induction of Middle Cerebral Artery Occlusion (MCAO)}

Focal cerebral ischemia was performed according to modified method of Longa et al. (1989). In brief, rats were anesthetized by thiopental sodium at dose of $50 \mathrm{mg} \mathrm{kg}^{-1}$ BW. The right common carotid artery and the right external carotid artery were exposed through a ventral midline neck incision and were ligated proximally. A silicone coated nylon monofilament (4-0) suture (USS DGTM sutures; Tyco Healthcare group LP, Connecticut, USA) with its tip rounded by heating near a flame was inserted through an arteriectomy in the common carotid artery just below the carotid bifurcation and then advanced into the internal carotid artery approximately $17-18 \mathrm{~mm}$ distal to the carotid bifurcation until a mild resistance was felt. Occlusion of the origins of the anterior cerebral artery, the middle cerebral artery and the posterior communicating artery was thereby achieved. Then, the wound was sutured, the rats were returned to their cages with free access to food and water. The incision sites were infiltrated with $10 \%$ PovidoneIodine Solution for anti-septic postoperative care.

\subsection{Behavioral Studies}

Behavior testing of all animals has been conducted every 7 days by blinded and trained observer. The animals has been assessed the changes of various behaviors using various test as described following; the assessment of the assessment of cognitive function (Morris water maze), the assessment of neurological score and the assessment of sensory function thermal stimulation (hot plate test).

\subsection{Assessment of Cognitive Function}

Animals were tested spatial memory by the water maze test (Morris et al., 1982). The apparatus was a pool with $170 \mathrm{~cm}$ diameter filled up with tap water for $40 \mathrm{~cm}$ deep and the water surface was covered with nontoxic powder. The pool was divided into four quadrants and the removable escape platform was placed in the center on one quadrant below the water level. For animals, the location of the platform was invisible and it remained there throughout the training. The animals must memorize the environment cues to locate the platform. Each animal was placed in the water in the starting quadrant and allowed to swim until it found and climbed onto the platform. The time for animal to reach the hidden platform was recorded as escape latency or acquisition time.

\subsection{Neurological Deficit Score}

All the animals were subjected to neurological evaluation by using 6-points postural reflex test according to the method of Bederson et al. (1986) and SchmidElsaesser et al. (1998). The deficit was graded from 0-5 as follow: Grade 0. No spontaneous activity; Grade 1:
Spontaneous circling; Grade 2: Circling if pulled by tail; Grade 3: Lowered resistance to lateral push without circling; Grade 4: Contralateral forelimb flexion; Grade 5: No apparent deficit.

\subsection{The Assessment of Sensory Function to Thermal Stimulation using Hot Plate Test}

The hot-plate test for rat were used to measure the latency of forepaw withdrawal response to thermal stimulation. The hot plate was held at $50^{\circ} \mathrm{C}$ and the test will be ended when the rat shakes or licks one of the paws or jumps. This test was applied 5 minute after the end of mechanical stimulation.

\subsection{Histological Procedure}

Following anesthesia with sodium pentobarbital (50 $\mathrm{mg} \mathrm{kg} \mathrm{BW}{ }^{-1}$ ), fixation of the brain was carried out by transcardial perfusion with fixative solution containing $4 \%$ paraformaldehyde in $0.1 \mathrm{M}$ phosphate buffer $\mathrm{pH} 7.3$. The brains were removed after perfusion and stored over a night in a fixative solution that used for perfusion. Then, they were infiltrated with $30 \%$ sucrose solution for approximately $4^{\circ} \mathrm{C}$. The specimens were frozen rapidly and $30 \mu \mathrm{M}$ thick sections were cut on cryostat. They were rinsed in the phosphate buffer and picked up on slides coated with $0.01 \%$ of aqueous solution of a high molecular weight poly L-lysine.

\subsection{Nissl Staining}

The duplicate coronal sections of brains were stained with $0.75 \%$ cresyl violet, dehydrated through graded alcohols $(70,95,100 \% 2 \times)$, placed in xylene and mounted using DPX.

\subsection{Morphological Analysis}

Five coronal sections of each rat in each group were studied quantitatively. Neuronal counts in hippocampus were performed by eye using a $40 \times$ objective with final field $255 \mu \mathrm{m} 2$ according to the following stereotaxic coordinates: AP-4.8 mm, lateral $\pm 2.4-6 \mathrm{~mm}$ and depth 3$8 \mathrm{~mm}$. The observer was blind to the treatment at the time of analysis. Viable stained neurons were identified on the basis of a stained soma with at least two visible processes. Counts were made in five adjacent fields and the mean number extrapolated to give the total number of neurons per $255 \mu \mathrm{m}^{2}$. All data were represented as number of neurons per $255 \mu^{2}$.

\subsection{Statistical Analysis}

Data were presented as mean \pm Standard Error of Mean (SEM). The analysis was performed using oneway Analysis of Variance (ANOVA), followed by LSD test. All statistical results were considered significant at p-value $<0.05$. 


\section{RESULTS}

\subsection{Effect of Mulberry Fruits Extract in Animal Model of Stroke in Morris Water Maze Test}

Cognitive deficit is commonly clinical sign in stroke. In this study examined the effect of mulberry fruit extract on spatial memory both healthy condition and stroke condition, after induce middle cerebral artery occlusion. Data were recorded escape latency and retention time. In healthy condition, the results showed significant changes in escape latency and retention times in rats which received either vitamin $\mathrm{C}$ or Aricept ${ }^{\circledR}$.Moreover, mulberry fruit significantly decreased escape latency time in dose $2,10 \mathrm{mg}$ $\mathrm{kgBW}^{-1}$ in single dose administration, 7 days administration.(p-value $<0.05$, all; compared with vehicle treated groups) (Fig. 1-2).

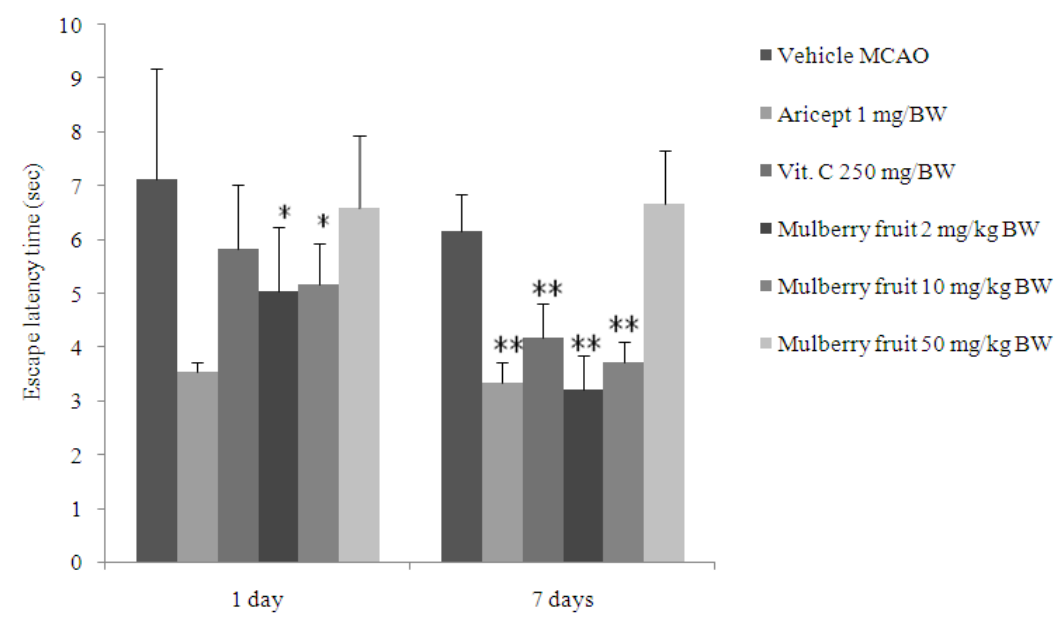

Fig. 1. The effect of mulberry fruit powder on escape latency time in healthy condition. Rats were treated with vehicle, Aricept ${ }^{\circledR}$, vitamin $\mathrm{C}$ or the mulberry fruit extract at various doses ranging from 2,10 and $50 \mathrm{mg} \mathrm{kg}^{-1} \mathrm{BW}$ via oral route for 7 days, then they were determined escape latency time in Morris water maze test. The test was performed within 30 minutes after the substance administration. Data are present as mean \pm SEM $(n=6 /$ group $) *$ p-value $<0.05$ compared with vehicle treated group, $* *$ p-value $<0.01$ and $* * *$ p-value $<0.001$ compared with vehicle treated group

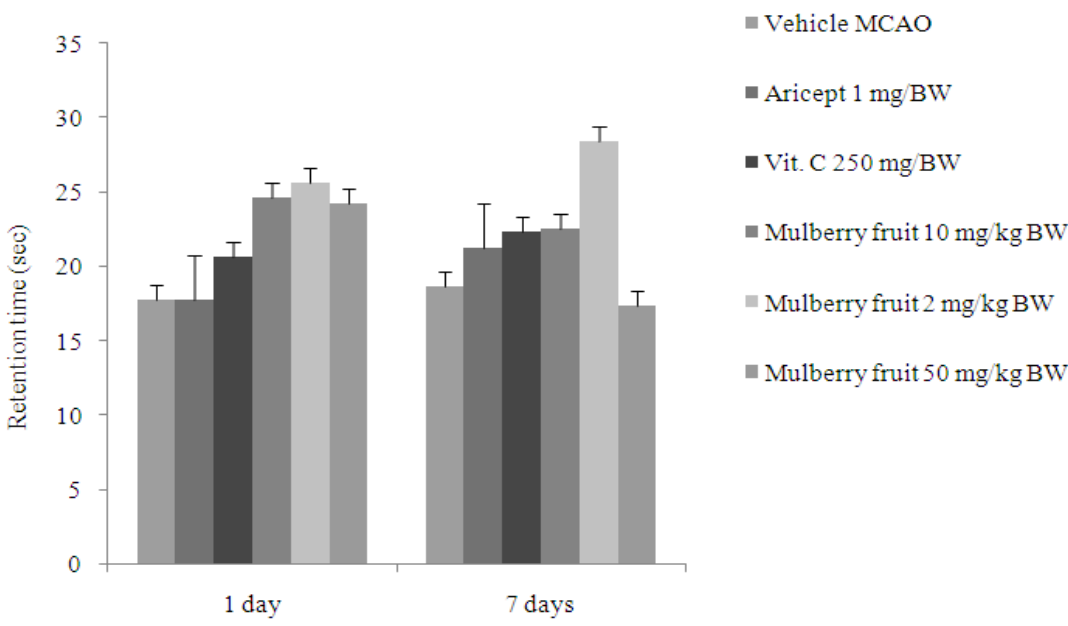

Fig. 2. The effect of mulberry fruit powder on retention time in healthy condition. Rats were treated with vehicle, Aricept ${ }^{\circledR}$, vitamin $\mathrm{C}$ or the mulberry fruit extract at various doses ranging from 2,10 and $50 \mathrm{mg} \mathrm{kg}^{-1} \mathrm{BW}$ via oral route for 7 days, then they were determined retention time in Morris water maze test. Data are present as mean \pm SEM $(n=6 /$ group $) * p$-value $<0.05$ compared with vehicle treated group, ${ }^{* *} \mathrm{p}$-value $<0.01$ and $* * * \mathrm{p}$-value $<0.001$ compared with vehicle treated group 


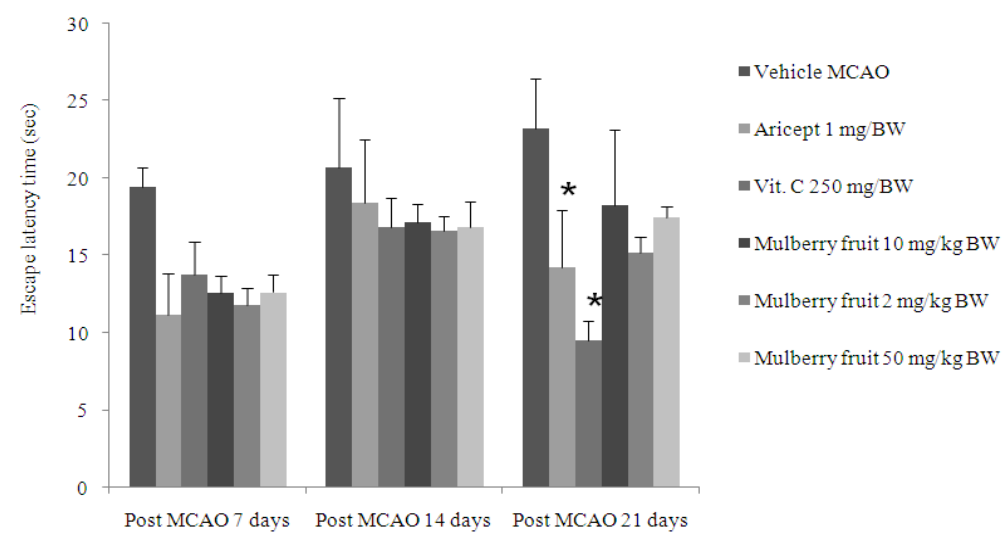

Fig. 3. The effect of mulberry fruit powder on escape latency time in stroke condition. Rats were treated with vehicle, Aricept ${ }^{\circledR}$, vitamin $\mathrm{C}$ or the mulberry fruit extract at various doses ranging from 2,10 and $50 \mathrm{mgkg}^{-1} \mathrm{BW}$ via oral route for 21 days, then they were determined escape latency time in Morris water maze test every 7 days. The test was performed within 30 minutes after the substance administration. Data are present as mean \pm SEM $(n=6$ /group $) *$-value $<0.05$ compared with vehicle treated group, $* *$ p-value $<0.01$ and $* * *$ p-value $<0.001$ compared with vehicle treated group

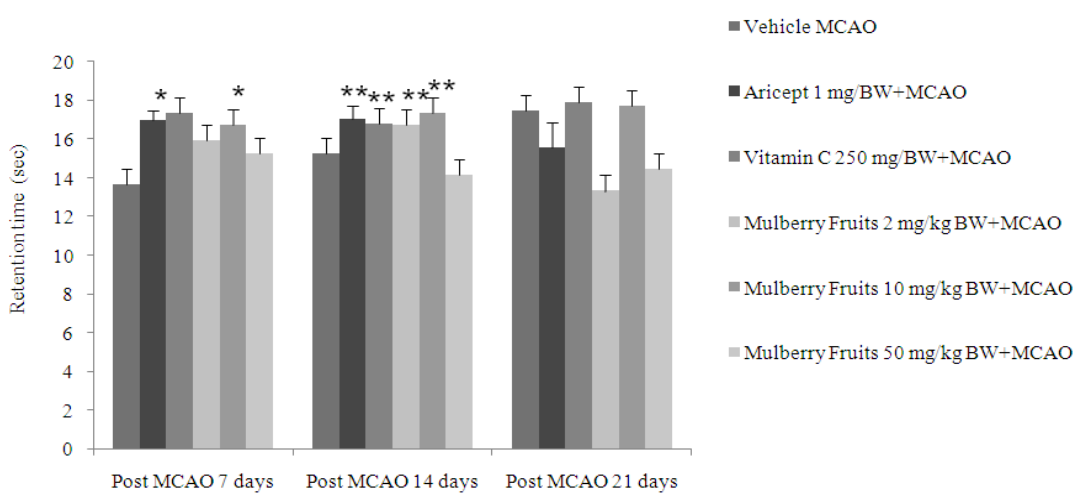

Fig. 4. The effect of mulberry fruit powder on retention time in stroke condition. Rats were treated with vehicle, Aricept ${ }^{\circledR}$, vitamin $C$ or the mulberry fruit extract at various doses ranging from 2,10 and $50 \mathrm{mgkg}^{-1} \mathrm{BW}$ via oral route for 21 days, then they were determined retention time in Morris water maze test every 7 days. Data are present as mean \pm SEM ( $n=6 /$ group) $* p$-value $<0.05$ compared with vehicle treated group, ${ }^{* *}$ p-value $<0.01$ and $* * *$ p-value $<0.001$ compared with vehicle treated group

In stroke condition, Vitamin $\mathrm{C}$, Aricept ${ }^{\circledR}$ and mulberry fruit powder at dose $2,10 \mathrm{mg} \mathrm{kgBW}^{-1}$ significantly increased the retention time in Morris water maze test after MCAO 14 days after MCAO when compared to the vehicle plus MCAO group (Fig. 3-4).

\subsection{Effect of Mulberry Fruit Extract on Neurological Score}

The effect of mulberry fruits powder on motor function was evaluated using neurological score as shown in Fig. 5. This test showed tendency of improvement in neurological score.

\subsection{Effect of Mulberry Fruit Powder on Thermal Stimulation}

The effect of mulberry fruits powder on motor function was evaluated using hot plate test as shown in Fig. 6.

\subsection{Effect of Mulberry Fruit Powder on Neuron Density}

Figure 7 shows that Aricept significantly increased the neuronal density in all sub regions of the hippocampus. Vitamin $\mathrm{C}$ significantly increased neuronal density only in CA1 (P-value $<0.05$ as compared with vehicle plus MCAO). Mulberry fruit powder at dose of 2, 10 and $50 \mathrm{mg}^{-1} \mathrm{~kg} \mathrm{BW}$ could increase the neuronal density in CA2 and CA3 whereas (P-value $<0.05$ as compared with vehicle plus MCAO). 
Pratchaya Kaewkaen et al. / American Journal of Agricultural and Biological Sciences 7 (3) (2012) 322-329

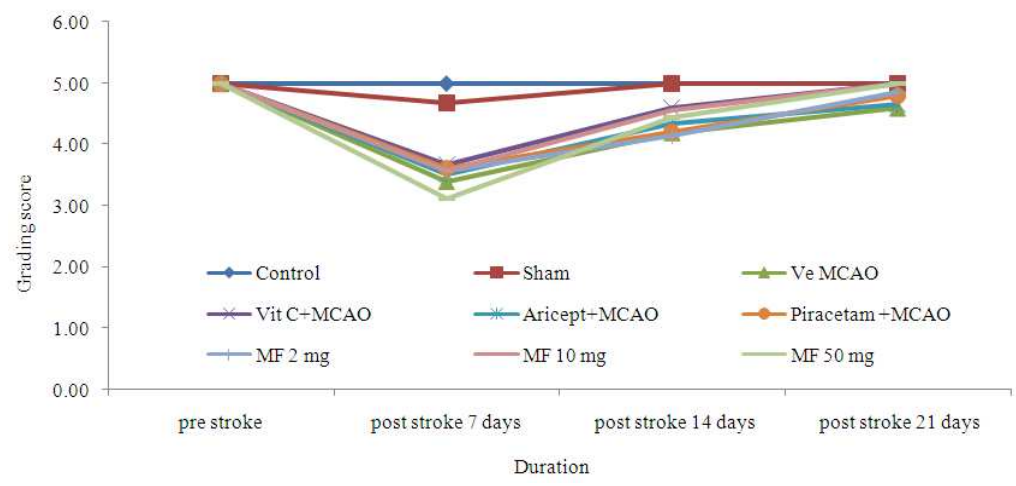

Fig. 5. The effect of mulberry fruit powder on neurological deficit score. Rats were treated with vehicle, Aricept ${ }^{\circledR}$, vitamin $C$ or the mulberry fruit extract at various doses ranging from 2,10 and $50 \mathrm{mg} \mathrm{kg}^{-1} \mathrm{BW}$ via oral route for 21 days. Data are present as mean \pm SEM $(n=6$ /group $) *$ p-value $<0.05$ compared with vehicle treated group. $*$ p-value $<0.05$ compared with vehicle treated group, $* *$ p-value $<0.01$ and $* * *$ p-value $<0.001$ compared with vehicle treated group

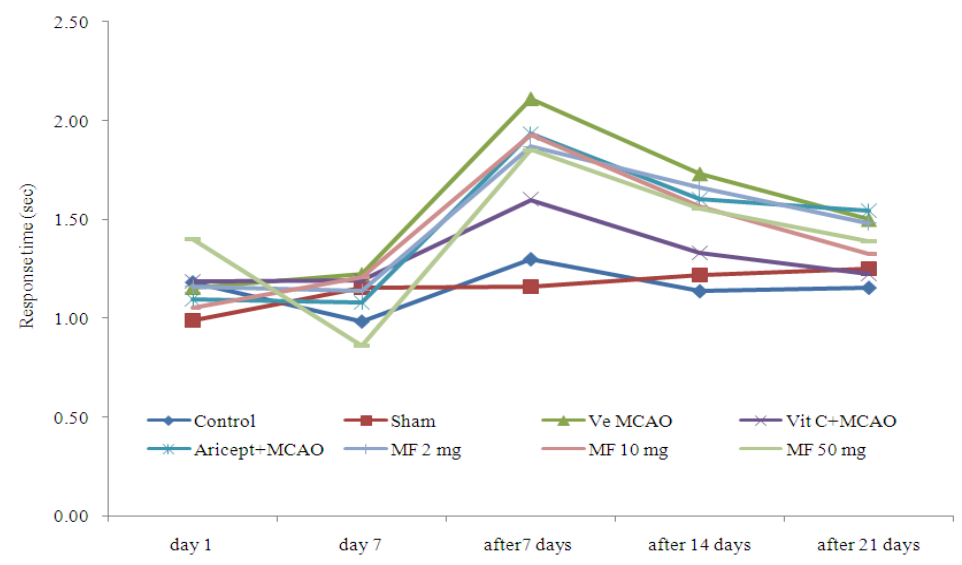

Fig. 6. The effect of mulberry fruit powder on hot plate test. Rats were treated with vehicle, Aricept ${ }^{\circledR}$, vitamin $\mathrm{C}$ or the mulberry fruit extract at various doses ranging from 2,10 and $50 \mathrm{mg} \mathrm{kg}^{-1} \mathrm{BW}$ via oral route for 21 days, then they were determined retention time in Morris water maze test every 7 days. Data are present as mean \pm SEM $(n=6 /$ group $) * p$-value $<0.05$ compared with vehicle treated group, ${ }^{* *}$ p-value $<0.01$ and $* * *$ p-value $<0.001$ compared with vehicle treated group

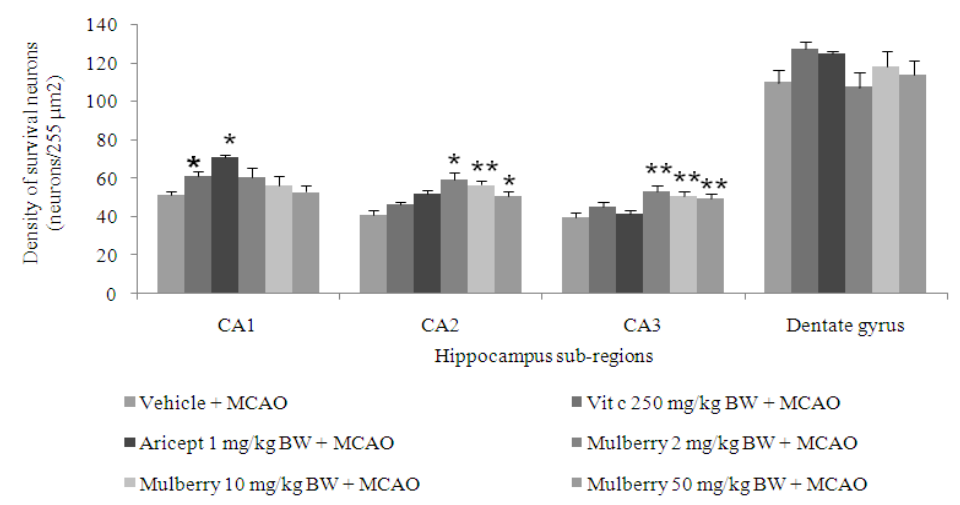

Fig. 7. The effect of mulberry fruit powder on neurons density in various subregions of hippocampus. Values given are the mean \pm S.E.M. $(n=6)$ P-value $<.05$ as compared with vehicle plus MCAO. Data are present as mean \pm SEM $(n=6 /$ group $) * p-$ value $<0.05$ compared with vehicle treated group, ${ }^{* *} \mathrm{p}$-value $<0.01$ and $* * *$ p-value $<0.001$ compared with vehicle treated group 


\section{DISCUSSION}

In this study, we have demonstrated the cognitive enhancing effect of Mulberry fruits powder via oral route administration in animal model of stroke. Our data showed that mulberry fruits decreased the escape latency time and increased retention time of Morris water maze test both health condition and stroke condition. The neurological score was increased after treated with mulberry fruit powder. The number of survival neurons was increases in hippocampus CA2, CA3 when compared with vehicle treated groups.

Animal model of ischemic stroke are procedure producing cerebral ischemia and the model can explain processes of pathophysiology of cerebral ischemia mechanism. Therefore a large portion of stroke research is conducted on animals. Most animal stroke models were developed to induce cerebral ischemia within the MCA territory in order to be relevant to clinical situation. The intraluminal suture MCAO in rats and in mice is the most frequently used model. This model is less invasive and easy to perform both permanent and transient ischemia in a controlled manner. Intraluminal suture MCAO model involves inserting a monofilament into the internal carotid artery and advancing until it blocks blood flow to MCA. This model provides reproducible MCA territory infarctions (involving both frontoparietal cortex and lateral caudoputamen) and allows reperfusion by retracting the suture. Depending on the shape, size and insertion length of the thread, the MCA can be either occluded selectively or in branches of internal carotid artery and the common carotid artery.

Learning has been defined as the process acquiring the knowledge while memory is the retention of the acquired knowledge that can be retrieved (Kupfermann 1993). Our results showed that under normal condition, mulberry fruit powder could exert its influence on both escape latency and retention time. Therefore, it indicated that it could influence on both learning acquisition, consolidation and retrieval processes. Since the pattern of changes of escape latency induced by mulberry fruit powder was similar to those of Aricept and vitamin C, we suggested that mulberry fruit powder might exert its effect to facilitate acquisition phase partly via the alteration of neurotransmitter such as acetylcholine and via its antioxidant effect.

Mulberries (Morus alba Linn.) are anthocyanins rich fruits, which are a traditional Chinese medicine. The major anthocyanins identified in the fruit extract are cyanidin 3glucoside and cyanidin 3-rutinoside (Suh et al., 2003). Recently, mulberry fruit has been reported to possess medicinal benefit, such as potent anti-oxidant activity (Chen et al., 2005; Kang et al., 2006), anti-inflammation (Kim and Park, 2006), anti-thrombotic (Yamamoto et al., 2006). Moreover, mulberry exhibit inhibitory effect on the migration and invasion of a human lung cancer cell line (Chen et al., 2005). Therefore, exploring the diverse bioactivities of mulberry fruit powder can be exploited pharmacologically associated with stroke.

\section{CONCLUSION}

Therefore, all results in this study suggested that mulberry fruit had high potential to be served as the functional food to protect against memory impairment and neurodegeneration induced by cerebral ischemia. However, precise underlying mechanism and the clinical trial study are still required before the implementation.

\section{ACKNOWLEDGEMENT}

This study was supported by the Integrative Complementary Alternative Medicine Research Group, Faculty of Medicine, Thailand and The Queen Sirikitti Department of Sericulture, Ministry of Agriculture and cooperative, Bangkok, Thailand.

\section{REFERENCES}

Bederson, J.B., L.H. Pitts, M. Tsuji, M.C. Nishimura and R.L. Davis et al., 1986. Rat middle cerebral artery occlusion: Evaluation of the model and development of a neurologic examination. Stroke, 17: 472-476. PMID: 3715945

Brainin, M., Y. Teuschl and L. Kalra, 2007. Acute treatment and long-term management of stroke in developing countries. Lancet Neurol., 6: 553-561. PMID: 17509490

Cada, A., J.C.D.L. Torre and F. Gonzalez-Lima, 2000. Chronic cerebrovascular ischemia in aged rats: Effects on brain metabolic capacity and behavior. Neurobiol. Aging, 21: 225-233. PMID: 10867207

Chen, C.C. L.K. Liu, J.D. Hsu, H.P. Huang and M.Y. Yang et al., 2005. Mulberry extract inhibits the development of atherosclerosis in cholesterol-fed rabbits. Food Chem., 91: 601-607. DOI: 10.1016/j.foodchem.2004.06.039

Dugo, P., L. Mondello and G. Errante, 2001 Identification of anthocyanins in berries by narrow bore high performance liquid chromatography with electrospray ionization detection. J. Agric. Food Chem., 49: 3987-3992. 
Kang, T.H., J.Y. Hur, H.B. Kim, J.H. Ryu and S.Y. Kim, 2006. Neuroprotective effects of the cyanidin-3-O$\beta$-d-glucopyranoside isolated from mulberry fruit against cerebral ischemia. Neurosci. Lett., 391: 122126. DOI: 10.1016/j.neulet.2005.08.053

Kim, A.J. and S. Park, 2006. Mulberry extract supplements ameliorate the inflammation-related hematological parameters in carrageenan-induced arthritic rats. J. Med. Food, 9: 431-435. PMID: 17004912

Kupfermann, I., 1993. Learning and Memory, Principles of Neural Sciences. 1st Edn., Prentice Hall International, London.

Lazze, M.C., M. Savio, R. Pizzala, O. Cazzalini and P. Perucca et al., 2004. Anthocyanins induce cell cycle perturbations and apoptosis in different human cell lines. Carcinogenesis, 25: 1427-1433. DOI: 10.1093/carcin/bgh138

Longa, E.Z., P.R. Weinstein, S. Carlson and R. Cummins, 1989. Reversible middle cerebral artery occlusion without craniectomy in rats. Stroke, 20: 84-91. DOI: 10.1161/01.STR.20.1.84

Meyer, C., J. Blissett and C. Oldfield, 2001. Sexual orientation and eating psychopathology: the role of masculinity and femininity. Int. J. Eat. Disord., 29: 314-318. PMID: 11262511

Mitcheva, M., H. Astroug, D. Drenska, A. Popov and M. Kassarova, 1993. Biochemical and morphological studies on the effects of anthocyans and vitamin $E$ on carbon tetrachloride induced liver injury. Cell Mol. Biol., 39: 443-448. PMID: 8329983

Morris, R.G., P. Garrud, J.N. Rawlins and J. O'Keefe, 1982. Place navigation impaired in rats with hippocampal lesions. Nature, 297: 681-683. PMID: 7088155
Park, C.H., Y.J. Lee, S.H. Lee, S.H. Choi and H.S. Kim et al., 2000. Dehydroevodiamine. $\mathrm{HCl}$ prevents impairment of learning and memory and neuronal loss in rat models of cognitive disturbance. J. Neurochem., 74: 244-253. PMID: 10617126

Raghuveer, C. and R.V. Tandon, 2009. Consumption of functional food and our health concern. Pak. J. Physiol., 5: 76-83.

Roman, G.C., P. Sachdev, D.R. Royall, R.A. Bullock and J.M. Orgogozo et al., 2004. Vascular cognitive disorder: A new diagnostic category updating vascular cognitive impairment and vascular dementia. J. Neurol. Sci., 226: 81-87. PMID: 15537526

Schmid-Elsaesser, R., S. Zausinger, E. Hungerhuber, A. Baethmann and H.J. Reulen, 1998. A critical reevaluation of the intraluminal thread model of focal cerebral ischemia: Evidence of inadvertent premature reperfusion and subarachnoid hemorrhage in rats by laser-doppler flowmetry. Stroke, 29: 21622170. DOI: 10.1161/01.STR.29.10.2162

Suh, H.J., D.O. Noh, C.S. Kang, J.M. Kim and S.W. Lee, 2003. Thermal kinetics of color degradation of mulberry fruit extract. Nahrung, 47: 132-135. PMID: 12744293

Tsuda, T., K. Majumder and K.K. Linask, 1998. Differential expression of flectin in the extracellular matrix and left-right asymmetry in mouse embryonic heart during looping stages. Dev. Genet., 23: 203-214. PMID: 9842715

Yamamoto, Y., T. Fukushima, Y. Suna, N. Ishii and A. Saeki et al., 2006. Photoconductive coaxial nanotubes of molecularly connected electron donor and acceptor layers. Science, 314: 1761-1764. DOI: 10.1126/science. 1134441 